\title{
FRECUENCIA DE HALLAZGOS RADIOGRÁFICOS EN LAS RADIOGRAFÍAS PANORÁMICAS DE PACIENTES ATENDIDOS EN LA UNIVERSIDAD SANTO TOMÁS: PERÍODO 2010 - 2012
}

\author{
${ }^{1}$ Silvia Juliana Rueda Hernández, ${ }^{1}$ Edwin Yesid Gallardo Suárez, ${ }^{1}$ Erica Natalia Villamizar Caballero, ${ }^{1}$ Mariana Díaz Gómez, \\ ${ }^{2}$ Sandra Juliana Rueda Velásquez \\ ${ }^{1}$ Odontólogo U. Santo Tomás, Colombia \\ ${ }^{2}$ Especialista en Patología Oral y Medios Diagnósticos U. del Bosque, docente U. Santo Tomás, Colombia
}

Autor responsable de correspondencia: Silvia Juliana Rueda Hernández.

Correo electrónico: silvia.rueda@ustabuca.edu.co

\begin{abstract}
RESUMEN
Objetivo: Determinar la frecuencia de hallazgos radiográficos en las radiografias panorámicas de los pacientes que acudieron a la Universidad Santo Tomás en el período 2010-2012.

Materiales y métodos: Se realizó un estudio observacional descriptivo retrospectivo con fuentes secundarias que evaluó un total de 766 radiografias que cumplieron con los criterios de elegibilidad, los investigadores de este estudio realizaron una estandarización de hallazgos radiográficos y elaboraron un formato que facilitó el registro de la información.

Resultados: De las 766 radiografias evaluadas 457 (59,7\%) pertenecían a mujeres, las lesiones más prevalentes se encontraron en maxilar superior, siendo las radiolucidas las más comunes con $284(37,1 \%)$ casos, dentro de las anomalías dentales se encontró que la dilaceración fue la más frecuente $167(21,8 \%)$; otros hallazgos como reabsorción radicular 129 (6,8\%) fueron también evidentes.

Conclusiones: Las lesiones radiolucidas uniloculares periapicales, la dilaceración y la hipertrofia de cornetes fueron los hallazgos más frecuentes que se encontraron en este estudio. [Rueda SJ, Gallardo EY, Villamizar EN, Díaz M. Rueda SJ. Frecuencia de hallazgos radiográficos en las radiografias panorámicas de pacientes atendidos en la Universidad Santo Tomás: período 2010-2012. Ustasalud 2015;14:19-24]
\end{abstract}

Palabras clave: Radiografia panorámica, prevalencia, clínicas odontológicas.

\section{FREQUENCY OF RADIOGRAPHIC FINDINGS IN PANORAMIC RADIOGRAPHS OF PATIENTS ATTENDING AT UNIVERSIDAD SANTO TOMÁS: PERIOD 2010 - 2012}

\section{ABSTRACT}

Objective: to determine the prevalence of radiographic findings in the panoramic radiographs of patients who attending to Universidad Santo Tomás University since 2010-2012.

Methods: We executed a retrospective descriptive observational study that evaluated a total of 766 radiographs that met the eligibility criteria, the researchers made a standardization of radiographic findings and designed a format that registered the information and data.

Results: a total of 766 radiographs were evaluated, 457 (59.7\%) belonged to women, most of the findings were located in the upper jaw, and corresponded to radiolucent lesions with $284(37.1 \%)$ cases, among dental anomalies, laceration was the most frequent 167 (21.8\%); Other findings such as radicular resorption 129 (6.8\%) were also evident.

Conclusions: panoramic radiography allows an overview of the jaws and shows hidden pathologies that do not become manifest clinically.

Keywords: Panoramic radiography, prevalence, dental clinics.

Recibido para publicación: 3 de septiembre de 2015. Aprobado para publicación: 27 de octubre de 2015. 


\section{INTRODUCCIÓN}

Una de las radiografias extra orales más utilizadas en odontología y sus especialidades es la Radiografía Panorámica (RP), debido a la posibilidad que ofrece de obtener una gran cantidad de las estructuras faciales y bucales ${ }^{1-3}$. La radiografía panorámica es una ayuda diagnóstica que brinda al odontólogo una visión única del paciente, contemplando toda la arcada y las estructuras circundantes, los huesos faciales, cóndilos, partes del seno maxilar y complejos nasales ${ }^{4-7}$. La identificación de patologías bucales por medio de esta herramienta ha adquirido una importancia en el diagnóstico de patologías bucales no visibles clínicamente ${ }^{8-10}$.

El objetivo del diagnóstico radiográfico en odontología, es reproducir las complejas áreas anatómicas del esqueleto facial de la forma más detallada posible y sin superposiciones. No cabe duda que el diagnóstico mejora cuando el objeto por examinar se encuentra en varios planos espaciales en lugar de una sola vista ${ }^{11-13}$.

La RP permite observar la anatomía completa del área maxilofacial con baja dosis de radiación para el paciente. La indicación de esta técnica incluye evaluación de traumatismos, enfermedades extensas de los maxilares, dentición mixta y dientes retenidos ${ }^{14,15}$.

En la Facultad de Odontología de la Universidad Santo Tomás de Bucaramanga se atienden pacientes con diferentes tipos de anomalías y solo al momento de analizar la RP se conoce con certeza la presencia de algunas patologías maxilofaciales.

Una dificultad que presenta el odontólogo general es la falta de criterio al momento de analizar la RP, al entender que no solo debe ocuparse en analizar las estructuras dentales, sino que también debe observar todas las estructuras anatómicas que componen una RP y así realizar un buen diagnóstico e indicar la terapéutica adecuada; además, debe realizarse un apropiado estudio radiológico que permita incluir una visión completa; en efecto, varios estudios han evidenciado que es posible encontrar hallazgos radiográficos importantes en este tipo de exámenes ${ }^{15-17}$.

La falta de análisis por parte del odontólogo y el uso inadecuado de estas ayudas diagnósticas son los principales problemas en la consulta odontológica, lo cual se refleja en la falta de diagnósti$\mathrm{CO}^{18-21}$. La necesidad de identificar y caracterizar dichos hallazgos en las Clínicas Odontológicas de la Institución condujeron el propósito de la presente investigación, lo que justifica evaluar la totalidad de historias clínicas disponibles, correspondientes a individuos que acudieron a los servicios de odontología durante el período 2010-2012.

\section{MATERIALES Y MÉTODOS}

Se realizó un estudio observacional descriptivo retrospectivo con fuentes secundarias ${ }^{22}$, se llevo a cabo un muestreo no probabilístico a conveniencia, se tomó la totalidad de las radiografias panorámicas de las Clínicas Odontológicas de la Universidad Santo Tomás de los años 2010-2012, se preservó la identidad de los individuos y esta investigación se catalogó sin riesgo, según la Resolución $\mathrm{N}^{\circ} 008430$ de 1993 del Ministerio de Salud de Colombia; se tuvo en cuenta la anatomía normal, patologías y demás descubrimientos presentes. De esta manera se analizaron 766 radiografias que fueron consideradas según los criterios de elegibilidad. Solo se analizaron radiografias panorámicas que se encontraran en las historias clínicas debidamente diligenciadas y aprobadas por el docente supervisor del estudiante, desde el año 2010 a 2012. Se decidió descartar aquellas RP sin nitidez, ni contraste, con fallas de procesamiento y de pacientes con artefactos.

Dentro del procedimiento que se realizó para el análisis de las RP los investigadores fueron instruidos, capacitados por medio de una docente (SJEV), quien dirigía el trabajo de grado; y siguiendo un protocolo, bajo la ayuda de negatoscopios y de un instrumento de elaboración propia, se consignaron cada uno de los hallazgos (Instrumento disponible en: goo.gl/Tf6Mc4). También se consideraron aspectos de interés para el estudio como estructuras anatómicas, anomalías dentales y otros aspectos radiográficos que se pudiesen observar. Las variables que se tuvieron en cuenta fueron clasificadas en imágenes radiolúcidas, radiopacas y mixtas, uniluculares, multiloculares, multifocales; según su ubicación se tuvo en cuenta si eran periapicales, pericoronales, interradiculares y en hueso basal, también se observaron anomalías dentales, implantes, restos radiculares, dientes incluidos y la presencia de reabsorción radicular.

\section{Análisis estadístico}

El procesamiento estadístico de la información se hizo con el paquete estadístico Stata ${ }^{\circledR} 11$ (Stata Corp. 2009. Stata Statistical Software: Release 11. College Station, TX: Stata Corp LP.) y comprendió un análisis univariado donde se calcularon frecuencias y porcentajes para las variables cualitativas o categóricas; mientras que para las variables numéricas o cuantitativas se obtuvieron medidas de tendencia central y de dispersión. Para el análisis bivariado se ejecutó la prueba estadística de $\mathrm{chi}^{2}$ teniendo en cuenta una significancia estadística de $\mathrm{p} \leq 0,05$ con el fin de analizar la relación del género con los diferentes hallazgos radiográficos encontrados. 


\section{RESULTADOS}

El total de radiografias panorámicas evaluadas fue de 766 de las cuales 457 (59,7\%) pertenecían a mujeres. Las estructuras anatómicas que se tuvieron en cuenta para este estudio fueron: tabique nasal, cornetes, seno maxilar, cóndilo y corticales, dentro de ellas se evaluó si existía alguna anormalidad; se encontró que $474(61,9 \%)$ pacientes presentaron tabique nasal recto y $292(38,1 \%)$ tabique desviado, también se encontraron $419(54,7 \%)$ pacientes con cornetes hipertróficos y $347(45,3 \%)$ con cornetes normales.

En cuanto al seno maxilar $393(51,3 \%)$ pacientes presentaron simetría y $373(48,7 \%)$ presentaron seno maxilar asimétrico; respecto al análisis de cóndilo, se evidenciaron $721(94,1 \%)$ cóndilos simétricos y $45(5,9 \%)$ asimétricos.

Adicionalmente, se encontró que 721 (94,1\%) pacientes presentaron corticales definidas y 45 $(5,9 \%)$ indefinidas (figura 1$)$.

Sobre la frecuencia de lesiones óseas, tanto en maxilar superior como en maxilar inferior, se demostró que aquellas radiolucidas fueron las más comunes en ambos maxilares (tabla 1).

Con relación a las anomalías dentales y otros aspectos radiográficos, se observó que la dilaceración fue la más frecuente $167(21,8 \%)$ seguida de la reabsorción radicular 129 (16,8\%) (tabla 2$)$.

$\mathrm{Al}$ analizar los hallazgos radiográficos según el sexo no se observó ninguna diferencia estadísticamente significativa para el maxilar superior, mientras que para el inferior se encontró que las lesiones radiopacas fueron más comunes en hombres que en mujeres $(p<0,0001)$ al igual que las lesiones pericoronales $(p=0,02)$ (tabla 3$)$.
Tabla 1. Frecuencia de hallazgos radiográficos en hueso maxilar y mandibular

\begin{tabular}{lcc}
\hline \multicolumn{1}{c}{ Hallazgos } & $\begin{array}{c}\text { Frecuencia } \\
\text { en el } \\
\text { maxilar } \\
\text { superior } \\
\text { n (\%) }\end{array}$ & $\begin{array}{c}\text { Frecuencia } \\
\text { en el } \\
\text { maxilar } \\
\text { inferior } \\
\text { n (\%) }\end{array}$ \\
\hline Lesiones radiolucidas & $284(37,1)$ & $258(33,7)$ \\
Lesiones radiopacas & $26(3,4)$ & $35(4,6)$ \\
Lesiones mixtas & $24(3,1)$ & $31(4)$ \\
Lesiones uniloculares & $244(31,8)$ & $241(31,5)$ \\
Lesiones multiloculares & $51(6,7)$ & $53(6,9)$ \\
Lesiones multifocales & $13(1,7)$ & $9(1,2)$ \\
Lesiones pericoronales & $26(3,4)$ & $21(2,7)$ \\
Lesiones periapicales & $214(27,9)$ & $223(29,1)$ \\
Lesiones interradiculares & $66(8,6)$ & $43(5,6)$ \\
Lesiones en hueso basal & $63(8,2)$ & $82(10,7)$ \\
\hline
\end{tabular}

Tabla 2. Frecuencia de anomalías dentales y otros hallazgos en maxilar y mandíbula

\begin{tabular}{lc}
\hline \multicolumn{1}{c}{ Anomalías } & n (\%) \\
\hline Macrodoncia & $34(4,4)$ \\
Microdoncia & $30(3,9)$ \\
Fusión & $124(16,2)$ \\
Geminación & $13(1,7)$ \\
Raíces supernumerarias & $22(2,9)$ \\
Dilaceración & $167(21,8)$ \\
Concrescencia & $7(0,9)$ \\
Dientes supernumerarios & $14(1,8)$ \\
Implantes & $7(0,9)$ \\
Restos radiculares & $93(12,1)$ \\
Dientes incluidos & $107(14)$ \\
Reabsorción radicular & $129(16,8)$ \\
Global & $747(97,5)$ \\
\hline
\end{tabular}

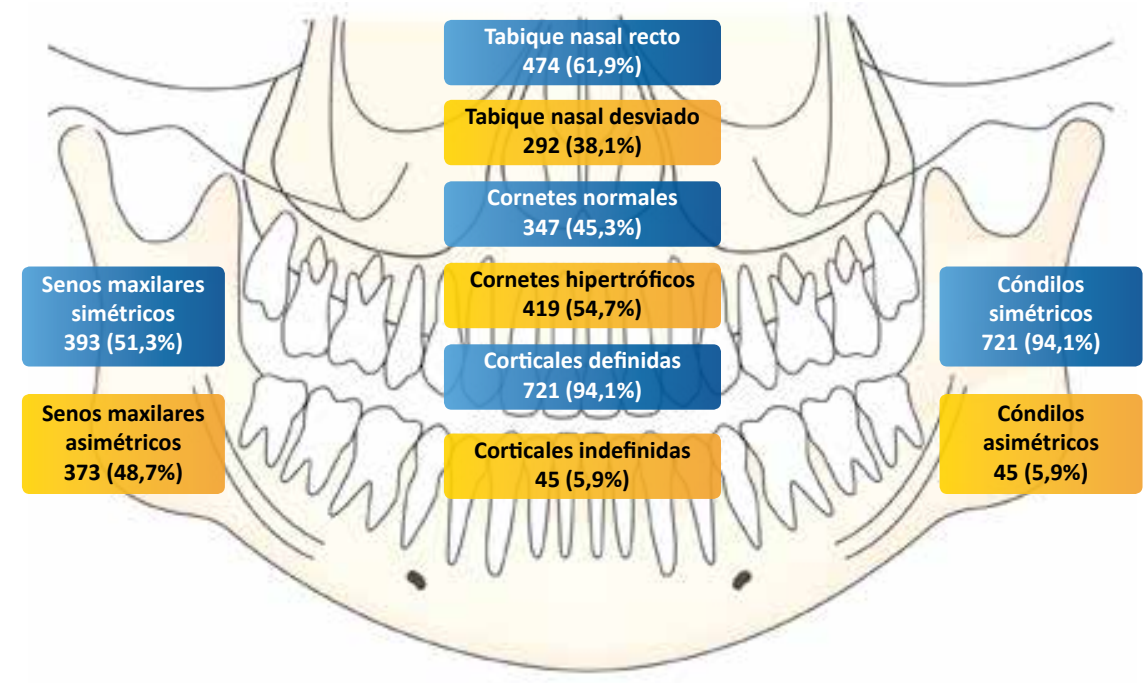

Figura 1. Principales anomalías de estructuras anatómicas 
Las fusiones dentales fueron más frecuentes en las mujeres que en los hombres $(p=0,05)$ al igual que los restos radiculares $(p=0,04)$, sin embargo, la concrescencia y la reabsorción radicular se presen- taron en más hombres que en mujeres con valores de significancia estadística de $\mathrm{p}=0,01$ y $\mathrm{p}=0,001$ respectivamente (tabla 4).

Tabla 3. Relación de lesiones radiográficas según el género

\begin{tabular}{lcccc}
\hline \multicolumn{1}{c}{ Variable } & $\begin{array}{c}\text { Global } \\
\mathbf{n}(\mathbf{\%})\end{array}$ & $\begin{array}{c}\text { Hombres } \\
\mathbf{n = 3 0 9} \mathbf{n}(\mathbf{\%})\end{array}$ & $\begin{array}{c}\text { Mujeres } \\
\mathbf{n = 4 5 7} \mathbf{n}(\%)\end{array}$ & $\mathbf{p}$ \\
\hline Maxilar superior & & & & \\
\hline Lesiones radiolucidas & $284(37)$ & $121(39,2)$ & $163(35,7)$ & 0,160 \\
Lesiones radiopacas & $26(3,39)$ & $12(1,9)$ & $14(3,1)$ & 0,270 \\
Lesiones mixtas & $24(3,13)$ & $12(3,9)$ & $12(2,6)$ & 0,160 \\
Lesiones uniloculares & $244(31,8)$ & $105(34)$ & $139(30,4)$ & 0,150 \\
Lesiones multiloculares & $51(6,65)$ & $20(6,5)$ & $31(6,8)$ & 0,430 \\
Lesiones multifocales & $13(1,69)$ & $4(1,3)$ & $9(2)$ & 0,250 \\
Lesiones pericoronales & $26(3,39)$ & $12(3,9)$ & $14(3,1)$ & 0,270 \\
Lesiones periapicales & $214(27,93)$ & $90(29,1)$ & $124(27,1)$ & 0,270 \\
Lesiones interradiculares & $66(8,61)$ & $31(10)$ & $35(7,7)$ & 0,120 \\
Lesiones hueso basal & $63(8,22)$ & $30(9,7)$ & $33(7,2)$ & 0,110 \\
\hline Maxilar inferior & & & & \\
\hline Lesiones radiolucidas & $258(33,6)$ & $103(33,3)$ & $155(33,9)$ & 0,430 \\
Lesiones radiopacas & $26(3,3)$ & $21(6,8)$ & $14(3,1)$ & 0,001 \\
Lesiones mixtas & $31(4)$ & $16(5,2)$ & $15(3,3)$ & 0,110 \\
Lesiones uniloculares & $241(31,4)$ & $102(33)$ & $139(30.4)$ & 0,220 \\
Lesiones multiloculares & $53(6,9)$ & $22(7,1)$ & $31(6,8)$ & 0,420 \\
Lesiones multifocales & $9(1,1)$ & $5(1,6)$ & $4(0,9)$ & 0,180 \\
Lesiones pericoronales & $21(2,7)$ & $13(4,2)$ & $8(1,8)$ & 0,020 \\
Lesiones periapicales & $223(29,1)$ & $94(30,4)$ & $129(28,2)$ & 0,250 \\
Lesiones interradiculares & $43(5,6)$ & $21(6,8)$ & $22(4,8)$ & 0,120 \\
Lesiones en Hueso basal & $82(10,7)$ & $28(9,2)$ & $52(11,8)$ & 0,110 \\
\hline
\end{tabular}

Tabla 4. Relación de anomalías, fracturas y otros hallazgos según el género

\begin{tabular}{lcccc}
\hline Variable & Global & $\begin{array}{c}\text { Hombre } \\
\mathbf{N}=\mathbf{3 0 9}\end{array}$ & $\begin{array}{c}\text { Mujer } \\
\mathbf{N}=\mathbf{4 5 7}\end{array}$ & $\mathbf{p}$ \\
\hline Macrodoncia & $34(4,4)$ & $13(4,2)$ & $21(4,6)$ & 0,410 \\
Microdoncia & $30(3,9)$ & $11(3,6)$ & $19(4,2)$ & 0,340 \\
Fusión & $124(16,1)$ & $58(18,8)$ & $66(14,4)$ & 0,050 \\
Geminación & $13(1,69)$ & $7(2,3)$ & $6(1,1)$ & 0,160 \\
Raíces supernumerarias & $22(2,87)$ & $11(3,6)$ & $11(2,4)$ & 0,170 \\
Dilaceración & $176(21,8)$ & $74(23,9)$ & $93(20,4)$ & 0,110 \\
Concrescencia & $7(0,9)$ & $6(1,9)$ & $1(0,2)$ & 0,010 \\
Dientes supernumerarios & $14(1,82)$ & $6(1,9)$ & $8(1,8)$ & 0,420 \\
Implantes & $7(0,91)$ & $2(0,6)$ & $5(1,1)$ & 0,280 \\
Restos radiculares & $93(12,1)$ & $35(14,6)$ & $48(19,5)$ & 0,040 \\
Dientes incluidos & $107(13,9)$ & $47(15,2)$ & $60(13,2)$ & 0,200 \\
Reabsorción radicular & $129(6,8)$ & $65(21)$ & $64(14)$ & 0,001 \\
\hline
\end{tabular}




\section{DISCUSIÓN}

El análisis adecuado de la radiografía panorámica permitió desarrollar el principal objetivo de este estudio el cual se basó en determinar la frecuencia de patologías y anomalías maxilares de los pacientes que asistieron a las Clínicas Odontológicas de la Universidad Santo Tomás en el período 2010-2012, los hallazgos más relevantes que este estudio identificó fueron las frecuencias de lesiones radiolucidas en maxilar superior, que correspondieron al 37,1\% y para el maxilar inferior al 33,7\%; dentro de las anomalías dentales la dilaceración y la reabsorción radicular fueron las más frecuentes con un $21,8 \%$ y un $16,8 \%$, respectivamente; al relacionar las lesiones radiográficas con la variable sexo se evidenció que para el maxilar superior no hubo diferencias estadísticamente significativas, pero sí para el inferior, donde una de las diferencias más notables fue aquella que estudió las lesiones radiopacas, puesto que los hombres presentaron más cantidad de lesiones que las mujeres $(p<0,001)$. Adicionalmente, también se pudo observar que 6 hombres presentaron concrescencia a diferencia de solo 1 mujer $(p=0,01)$.

Al igual que los resultados descritos en el estudio realizado por Rushton ${ }^{23}$, se puede confirmar la importancia de una radiografia cuando se está diagnosticando cualquier tipo de patología en un paciente, ratificando que la RP proporciona una visión completa y precisa de la cavidad bucal.

Según el estudio realizado en Medellín por Aguilar et al. ${ }^{24}$ se detectó una frecuencia de hallazgos radiográficos en el que el $96 \%$ presentó algún tipo de alteración o patología, como caries, cálculos, anomalías dentarias basándose en estructuras anatómicas; este trabajo se enfocó en lesiones óseas maxilares y mandibulares, donde se evidenció que las lesiones radiolucidas en el maxilar superior fueron las más comunes con un $37 \%$ de prevalencia, puede decirse, entonces, que existe una mayor frecuencia de lesiones radiolucidas en la población estudiada.

A partir de este estudio se destaca la necesidad de darle importancia a este tipo de ayuda diagnóstica, pues una de las limitaciones del trabajo fue la ausencia de la RP en algunas de las historias clínicas de la Institución, otro aspecto que dificultó la recolección de información fue la falta de nitidez en la imagen de las radiografias y la presencia de artefactos, donde se observa la necesidad del uso de equipos panorámicos digitales; se sugiere además que para futuros estudios los investigadores realicen un ejercicio de calibración en el que se pueda conocer precisamente la reproducibilidad inter e intra examinador; a pesar de lo anterior, en este trabajo, un tercer investigador con más experiencia
(SJRV) estuvo presente para resolver cualquier caso de discrepancia.

Se puede concluir que el hallazgo más frecuente fue la hipertrofia de cornetes y las lesiones radiolúcidas uniloculares de localización periapical.

Las mujeres presentaron mayor número de lesiones periapicales y los hombres pericoronales. Las anomalías dentales más prevalentes fueron en su orden: dilaceración, fusión, reabsorción radicular.

\section{REFERENCIAS}

1. Park J. The evaluation of digital panoramic radiographs taken for implant dentistry in the daily practice. Med Oral Patol Oral Cir Bucal. 2010;15(4):e663-6. doi:10.4317| medoral.15.e663

2. Meesa IR, Srinivasan A. Imaging of the oral cavity. Radiol Clin North Am. 2015;53(1):99-114. doi: 10.1016/j. rcl.2014.09.003

3. Whaites E, Drage N. Radiography and radiology for dental care professionals: Elsevier Health Sciences; 2013.

4. Cedeño G, Casasa A, Gurrola B. Comparación de las técnicas radiográficas periapical y panorámica en premolares. Rev Mex Odon Clín. 2007;1(10):10-6.

5. White SC, Pharoah MJ. Oral Radiology-E-Book: Principles and Interpretation: Elsevier Health Sciences; 2014.

6. MacDonald D. Oral and maxillofacial radiology a diagnostic approach: John Wiley \& Sons; 2011.

7. Martínez ME, Martinez BAM, Bruno IG. Anatomía normal en la radiografía panorámica. Rev Ateneo Argent Odontol. 2008;47(3):18-21.

8. Bahlis A, Mezzomo LA, Boeckel D, Costa NPd, Teixeira ER. Accuracy of periapical radiography, panoramic radiography and computed tomography for examining the mental foramen region. Revista Odonto Ciência. 2010;25(3):2827. doi: /10.1590/S1980-65232010000300012

9. Whaites E, Drage N. Essentials of dental radiography and radiology: Elsevier Health Sciences; 2013.

10. Alattar MM, Baughman RA, Collett WK. A survey of panoramic radiographs for evaluation of normal and pathologic findings. Oral Surg Oral Med Oral Pathol. 1980;50(5):472-8. doi: 10.1016/S0030-4220(80)80017-X

11. Thomson EM, Johnson ON. Essentials of dental radiography for dental assistants and hygienists. 9th ed: Pearson; 2012.

12. Angelopoulos C, Bedard A, Katz JO, Karamanis S, Parissis N. Digital panoramic radiography: An overview. Seminars in Orthodontics. 2004;10(3):194-203. doi: $10.1053 / \mathrm{j}$. sodo.2004.05.003

13. Zuluaga-López OH, Muñoz-Alzate LM. Evaluación clínica y radiográfica de 30 implantes dentales colocados en un servicio odontológico de posgrado. Rev. CES Odont. 2014;27(1):18-30.

14. Martínez M, Martínez B, Bruno I. Radiografia panorámica en la práctica dental: alcances y limitaciones. RAAO 2008;47(2):18-21.

15. Altug-Atac AT, Erdem D. Prevalence and distribution of dental anomalies in orthodontic patients. Am J Orthod Dentofacial Orthop. 2007;131(4):510-4. doi: 10.1016/j.ajodo.2005.06.027 
16. Langland OE, Langlais RP, Preece JW. Principles of dental imaging: Lippincott Williams \& Wilkins; 2002.

17. Arana-Fernández de Moya E, Buitrago-Vera P, Benet-Iranzo F, Tobarra-Pérez E. Tomografía computerizada: introducción a las aplicaciones dentales. RCOE 2006;11(3):31122.

18. Rushton V, Horner $\mathrm{K}$, Worthington $\mathrm{H}$. Routine panoramic radiography of new adult patients in general dental practice: relevance of diagnostic yield to treatment and identification of radiographic selection criteria. Oral Surg Oral Med Oral Pathol. 2002;93(4):488-95. doi: http:// dx.doi.org/10.1067/moe.2002.121994

19. Updegrave, W.J. The Role of Panoramic Radiology in Diagnosis. Oral Surg Oral Med Oral Pathol. 1966;22:49-57.

20. Díaz-Pérez R, Echaverry-Navarrete RA. Agenesia en dentición permanente. Rev. salud pública. 2009;11(6):961-9. doi: 10.1590/S0124-00642009000600012

21. Arreaza A, Lara M. Ateroma calcificado en carótida y radiografía panorámica: reporte de caso. Acta Odontológica Venezolana. 2011;49(3).
22. Grimes DA, Schulz KF. An overview of clinical research: the lay of the land. The Lancet 2002;359(9300):57-61. doi: 10.1016/S0140-6736(02)07283-5

23. Rushton MN, Rushton VE. A study to determine the added value of 740 screening panoramic radiographs compared to intraoral radiography in the management of adult ( $>$ 18 years) dentate patients in a primary care setting. J Dent. 2012;40(8):661-9. doi: 10.1016/j.jdent.2012.04.018

24. Aguilar O, Gallego C, Iriarte A, Quintero S. Prevalencia de hallazgos en radiografias panorámicas de rutina. Rev Nac Odontol. 2009;5:15-20.

\section{Correo electrónico de los autores}

Silvia Juliana Rueda H.: silviajruedah.od@hotmail.com Edwin Yesid Gallardo S.: edwin.gallardo@hotmail.com Sandra Juliana Rueda V.: sajuruve@hotmail.com

Erica Natalia Villamizar: thalia2906@hotmail.com Mariana Diaz Gomez: marianita_diaz82@hotmail.com

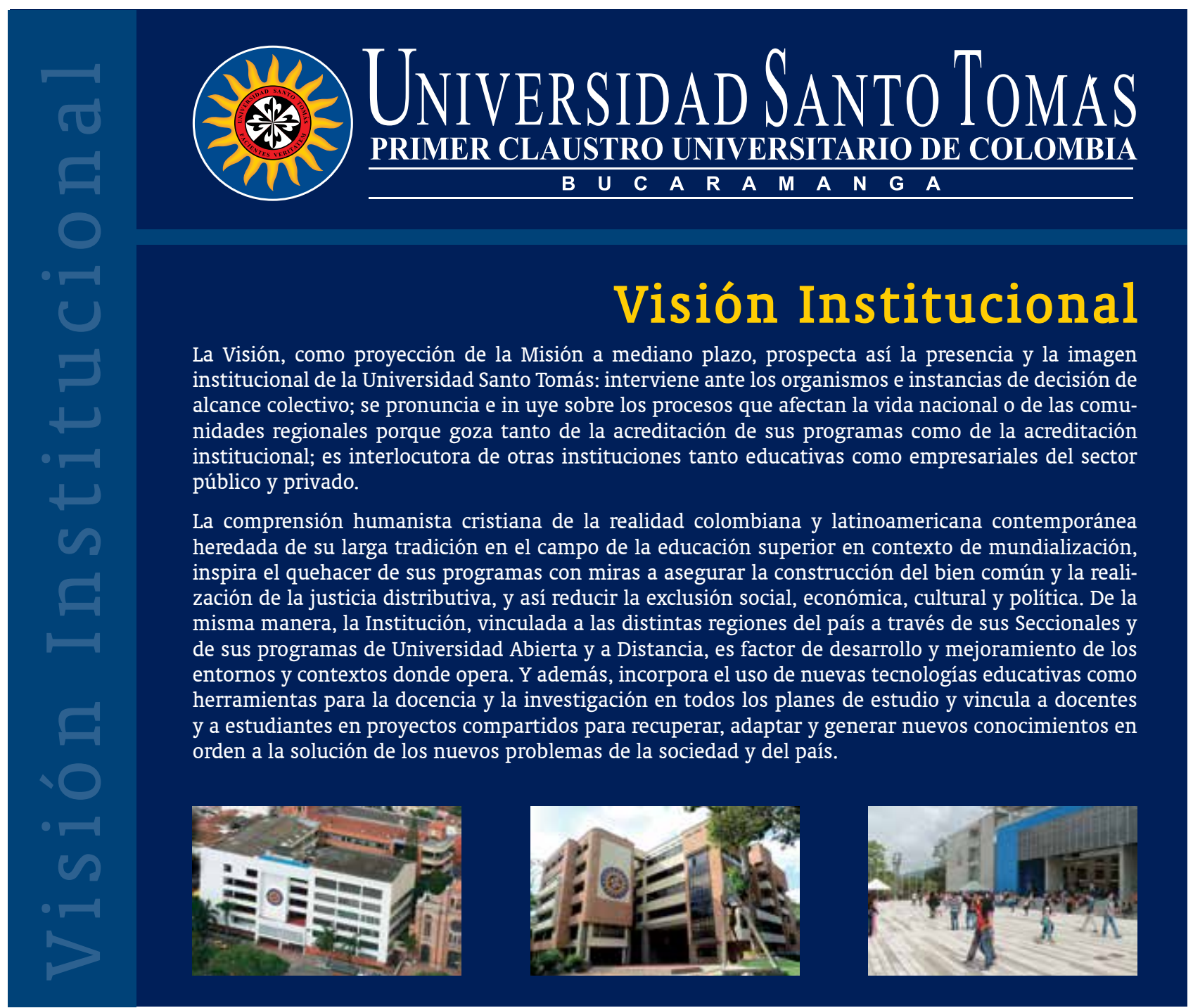

